\title{
Lösungsmöglichkeiten nach fehlgeschlagener Osteosynthese am distalen Oberarm/proximalen Unterarm
}

\author{
Christoph Josten, Bastian Marquaß
}

\section{Zusammenfassung}

Das Misslingen einer Osteosynthese führt zu einer vermehrten Belastung des Patienten und zu einem meist verlängertem krankheitsbedingten Ausfall der Erwerbstätigkeit. Die Gründe für ein Fehlschlagen sind häufig mannigfaltig und reichen von einer gestörten Vaskularisierung des Wundgebietes, Infektion, Instabilität der Frakturzone, Ausheilung in Fehlstellung, Implantatbruch bis hin $\mathrm{zu}$ einer verminderten Compliance des Patienten. Lösungskonzepte müssen daher individuell und ursachenbezogen entwickelt werden. Als Basisdiagnostik empfehlen sich konventionelle Röntgenaufnahmen. Bei spezieller Fragestellung sollten weitere bildgebende Verfahren wie CT oder Szintigraphie hinzugezogen werden.

\section{Distaler Humerus}

Distale Humerusfrakturen gehen in bis zu $40 \%$ der Fälle mit Begleitverletzungen einher [1] und etwa jede vierte Fraktur findet im Rahmen eines Polytraumas statt. Die Einteilung der Frakturen erfolgt entsprechend der AO-Klassifikation [8] und erlaubt so, neben prognostischen Aussagen auch eine reproduzierbare Vorgehensweise bei der Frakturversorgung.

Ergebnisse konservativer Therapieversuche aus der Vergangenheit lassen eine großzügige Indikationsstellung zur operativen Versorgung gerade bei intraartikulären Frakturen zu [2].

OP-JOURNAL 2004; 20: 196-200

(c) Georg Thieme Verlag KG Stuttgart · New York
Zur Versorgung von A-Frakturen gemäß der AO-Klassifikation ist meistens eine Schraubenosteosynthese ausreichend. Besonders bei epikondylären Abrissfrakturen ist eine Fragmentfixierung mit einer Schraubenosteosynthese sinnvoll. Aber auch im Bereich von unikondylären B-Verletzungen kann die Schraubenosteosynthese eventuell in Kombination mit einer Plattenosteosynthese angewandt werden.

Die radiale Platte, meistens eine RekoPlatte liegt dorsal an und wird soweit wie möglich an das Capitulum radii anmoduliert ohne das Gelenk zu tangieren. Dabei können auch von dorsal eingebrachte Schrauben Capitulumfrakturen fixieren. Die ulnare Platte wird entlang der Humeruskante anmoduliert und kann unter Identifikation des N. ulnaris um den Epicondylus ulnaris gebogen werden. Damit können dann auch lange 3,5mm-Kortikalisschrauben zur Fixierung von Trochleafragmenten eingesetzt werden.

Revisionseingriffe sind bei instabiler Versorgung, Materialbruch, sekundärer Dislokation, Infektion, Ankylose und Ausbildung einer Pseudarthrose notwendig. Das Ziel der Revision muss es sein, eine funktionelle und schmerzorientierte Verbesserung des Patienten zu erreichen. Eine Infektion ausgenommen, stehen als Verfahren die Reosteosynthese oder eine prothetische Versorgung zur Verfügung. Die Reosteosynthese sollte als Plattenosteosynthese durchgeführt werden, um eine möglichst hohe Stabilität der Frakturzone zu gewährleisten. Bei Vorliegen einer atrophischen Pseudarthrose ist auch die Interposition eines spongiösen Spans möglich. Aufwändig rekonstruktive Maßnahmen sind anspruchsvoll und können zur Durchführung eines Stufenkonzeptes im Rahmen mehrfacher Operationen führen [10].
Die Reosteosynthese des distalen Humerus erfolgt als Plattenosteosynthese zur maximalen Stabilisierung der Frakturzone. Bei komplexen Frakturen kann auch eine prothetische Versorgung als Revisionseingriff erfolgen.

\section{Olekranonfraktur}

Etwa 90\% aller Olekranonfrakturen werden durch ein direktes Trauma ausgelöst. Als Klassifikation hat sich die Fraktureinteilung nach Schatzker [9] durchgesetzt. Demnach werden 6 Subtypen anhand des Frakturverlaufes unterschieden. Bei gleichzeitig bestehender Radiusköpfchenluxation liegt eine Monteggia-Verletzung vor. Begleitverletzungen sind im Gegensatz zur distalen Humerusfraktur selten. $80 \%$ aller Olekranonfrakturen sind isolierte Verletzungen [5]. Im Wesentlichen stellt die operative Versorgung die Standardtherapie bei Olekranonfrakturen dar. Lediglich nichtdislozierte Frakturen bei älteren Personen können konservativ behandelt werden.

Bei einfachen Olekranonfrakturen ist die Zuggurtungsosteosynthese das Standardverfahren. Durch das dorsale Einbringen von zwei Kirschner-Drähten, welche die gegenseitige Kortikalis durchbohren sollten und Anlage einer Drahtcerclage als Achterschlinge, kommt es zur Umwandlung von Zug- in Druckkräfte, wodurch eine Kompression der Frakturenden erreicht wird. Bei gleichzeitiger Coronoidfraktur empfiehlt sich das Einbringen einer zusätzlichen Schraube zur Fixation.

Liegt eine Mehrfragmentfraktur vor, so ist aus Stabilitätsgründen eine dorsale Plattenosteosynthese vorzuziehen. Dabei wird die proximalste Schraube der anatomisch angepassten und vorgebogenen Platte etwa in einem $90^{\circ}$-Winkel zu den anderen Schrauben eingebracht und in der Spongiosa platziert. 


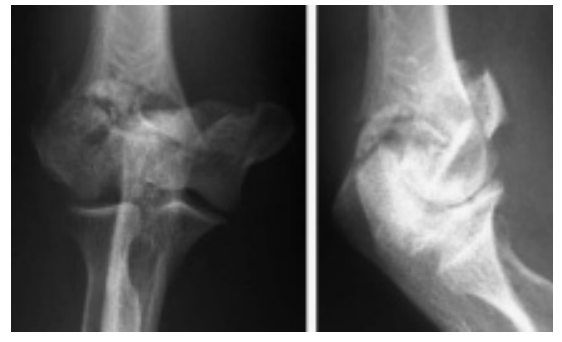

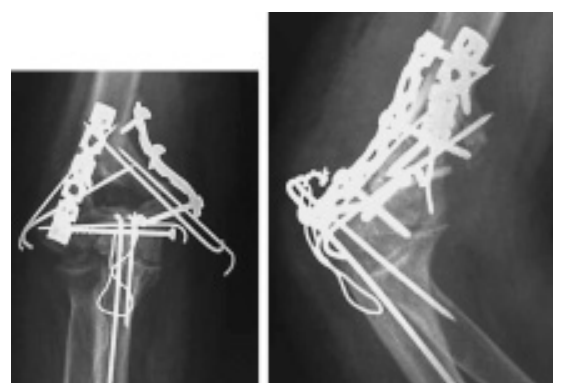
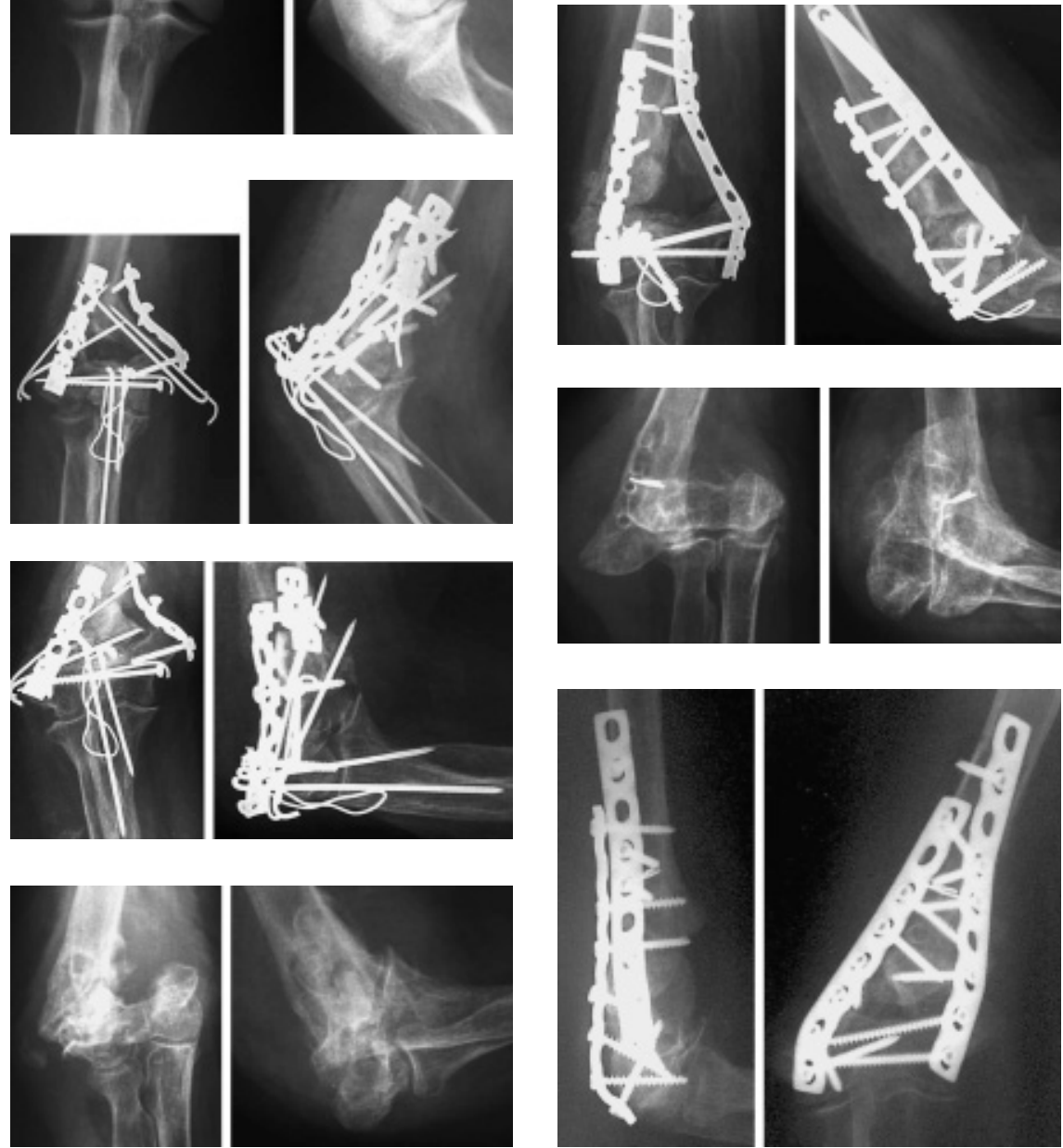

Abb.1 Die Bilder zeigen den Verlauf bei einer 70-jähriger Patientin mit initialer C3-Fraktur des distalen Humerus nach Sturz. Nach primärer Versorgung mit Platten- und Kirschner-Drahtosteosynthese kam es zur Ausbildung einer atrophen Pseudarthrose. Daraufhin erfolgte 9 Monate nach der primären Versorgung eine Reosteosynthese. Im weiteren Verlauf kam es zur erneuten Dislokation. Nach Materialentfernung Entschluss zur erneuten Plattenosteosynthese.

Komplikationen nach Olekranonfrakturen finden sich bei $14-25 \%$ der Patienten [5]. Klinisch stehen besonders Bewegungseinschränkungen und Schmerzen im Vordergrund. Diese werden nicht selten ausgelöst durch eine insuffiziente Osteosynthese, die Ausbildung einer Pseudarthrose oder eine nicht erkannte Monteggia-Verletzung. Seltener kommt es zu heterotopen Ossifikationen oder Nerven-Gefäß-Läsionen, die meistens das Versorgungsgebiet des N. ulnaris betreffen. Instabilitäten sind selten. Liegen sie dennoch vor, so häufig in Assoziation mit einer einhergehenden Kapsel-BandLäsion.

Bei Revisionseingriffen dominiert die Reosteosynthese. Hohe Stabilitätswerte reduzieren das Risiko einer Pseudarthrose. Daher ist auch am Olekranon die bedeutendste Art der Reosteosynthese die Plattenosteosynthese. Je nach Defekt und Ursache erfolgt die Kombination mit einer Spongiosaplastik.

Klinische Bewegungseinschränkungen und persistierende Schmerzen nach Olekranonosteosynthesen sind häufig auf insuffiziente Osteosynthesen zurückzuführen. Ein Revisionseingriff kann mit einer Spongiosaplastik kombiniert werden.
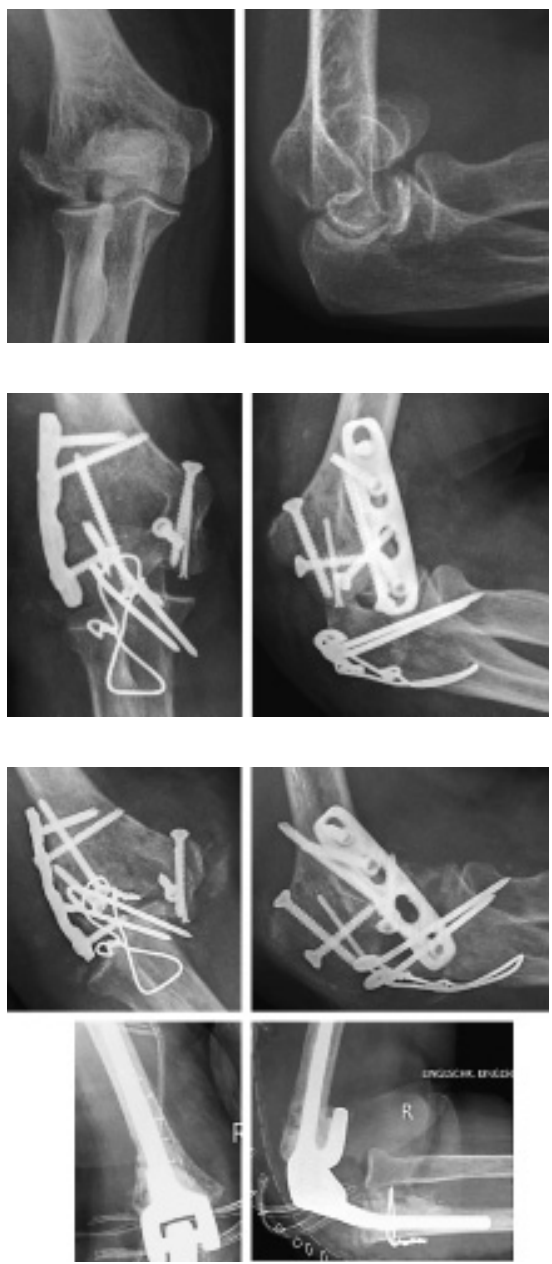

Abb.2 Bei der 68-Jährigen Patientin, ebenfalls mit C3-Verletzung nach Sturz, kam es nach Frakturversorgung mittels Plattenosteosynthese und Chevron-Osteotomie bei schwieriger Compliance der Patientin neben einer Dislokation zur Ausbildung einer Pseudarthrose. Aufgrund der erheblichen Gelenkdestruktion und einer deutlichen klinischen Beschwerdesymptomatik wurde die Indikation zur Prothesenimplantation gestellt.

\section{Radiusköpfchenfraktur}

Die Radiusköpfchenfraktur macht etwa 20-30\% aller Ellenbogenfrakturen aus. Ursache ist meistens ein direktes Trauma, wodurch es in bis zu 30\% zu Begleitverletzungen kommt. Die häufigsten sind Ruptur des Ligaments, Ruptur der Membrana interossea, Fraktur des Olekranons oder weiter distal der Handwurzeln. Die Einteilung erfolgt je nach Frakturtyp und Begleitverletzungen anhand der von McKee und Jupiter [7] modifizierten Mason-Klassifikation [6]. 

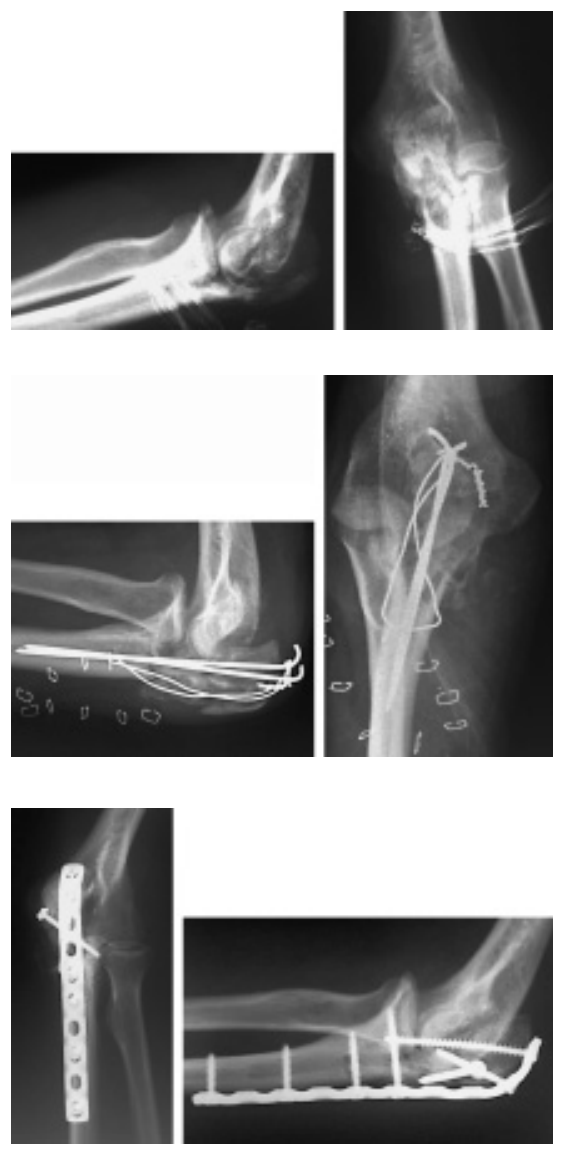

Abb. 3 Der Verlauf zeigt eine primäre Versorgung einer Olekranonmehrfragmentfraktur mit einer Zuggurtungsosteosynthese und Spongiosaplastik bei einem 20-jährigen Patienten. Es kam zur Ausbildung einer Pseudarthrose. Diese ging klinisch mit Bewegungsschmerz und deutlicher Bewegungseinschränkung einher. Intraoperativ zeigte sich eine Ankylosierung des proximalen Fragmentes mit der Trochlea. Nach Fragmentlösung wurde mit einer LCDC-Platte die Pseudarthrose stabilisiert und durch Einbringen einer interfragmentären Zugschraube zusätzlich komprimiert.

Liegen keine zusätzlichen Verletzungen vor, wird die Mason-I-Fraktur fast ausschließlich konservativ frühfunktionell behandelt. Eine relative OP-Indikation besteht bei Mason-II-Verletzungen in Abhängigkeit von Begleitverletzungen, Instabilität, Bewegungseinschränkungen sowie Alter und Anspruch des Patienten. Als Osteosyntheseverfahren bieten sich die Schraubenosteosynthese, die Aufrichtung und Retention durch Prevot-Nägel, die Fragment-/Radiusköpfchenresektion, seltener die Plattenosteosynthese und neuere Verfahren wie die Fixierung mit biodegradierbaren Stiften an. Der Behandlungsalgorithmus bei Mason-IIIund -IV-Frakturen ist unten stehend dargestellt.

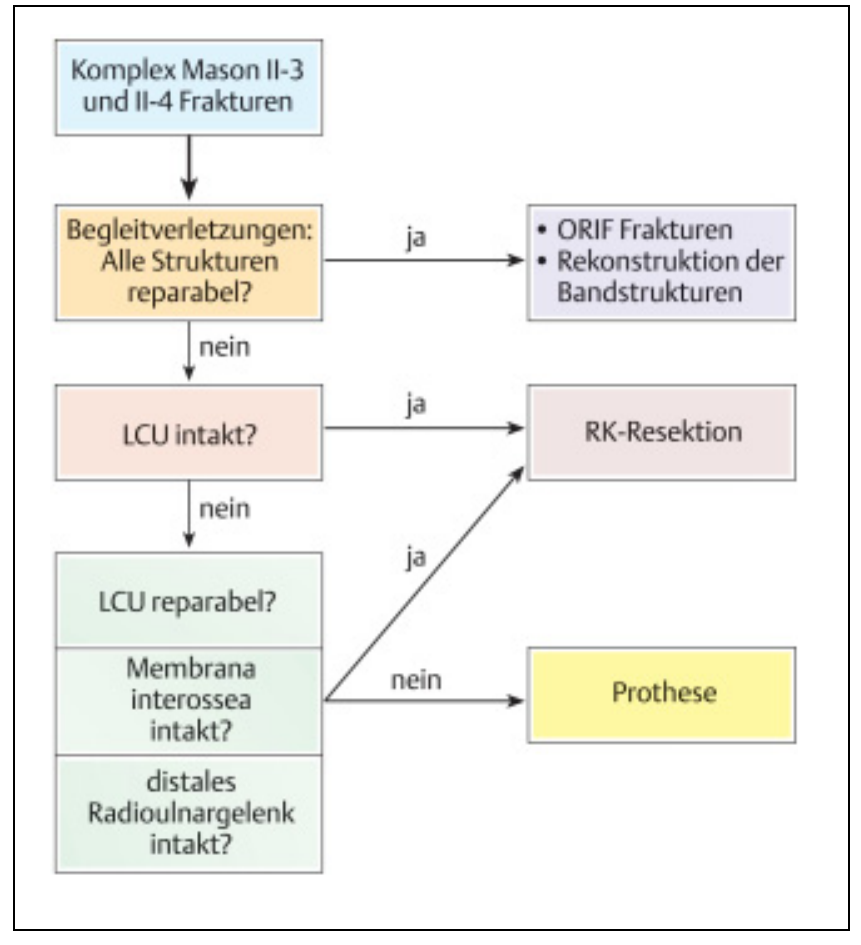

Abb. 4 Behandlungsalgorithmus bei Mason III/IVFrakturen; entnommen aus Josten $C$, Lill $\mathrm{H}$. Ellenbogenverletzungen 2002.

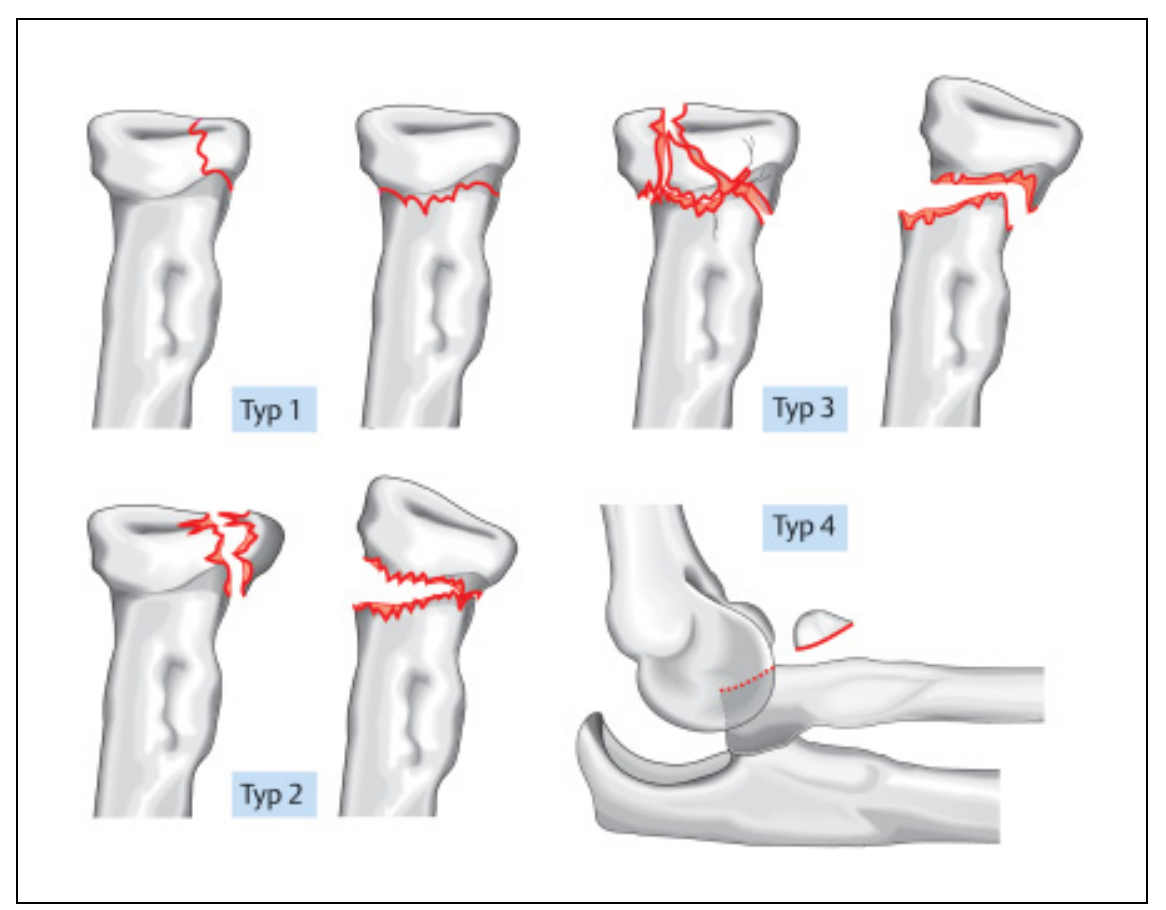

Abb.5 Einteilung der Radiusköpfchenfrakturen nach Mason; entnommen aus Josten C, Lill H. Ellenbogenverletzungen 2002.

An Komplikationen bei Radiusköpfchenfrakturen werden in bis zu $50 \%$ Bewegungseinschränkungen beobachtet. Diese finden sich in Abhängigkeit von dem Frakturtyp und von der Dauer der Immobilisation. Patienten, die länger als 10 Tage immobilisiert wurden, wiesen größere Bewegungsdefizite auf als $\mathrm{Pa}-$ tienten, die rascher funktionell behandelt werden konnten.

Instabilitäten treten bei intaktem KapselBand-Apparat nach einfachen Radiusköpfchenresektionen nicht auf. Erst bei komplexeren Frakturtypen wie Mason IV oder Essex-Lopresti-Verletzungen 

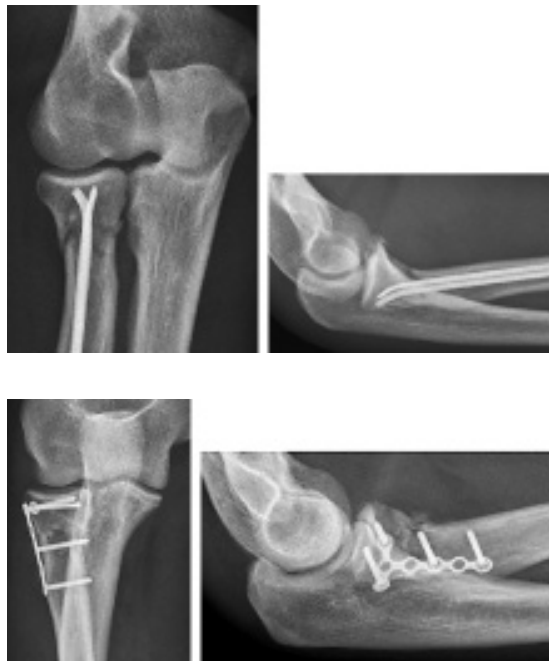

Abb.6 Bei dem 46-jährigen Patienten erfolgte die primäre Versorgung einer MasonII-Fraktur mit Prevot-Nägeln. Nach sekundärer Dislokation und Reosteosynthese mit einer Leibinger-platte kam es zur Frakturkonsolidierung.

kann es zur Instabilität kommen. Im Gegensatz zu den Olekranonfrakturen wird das Auftreten von heterotopen Ossifikationen in bis zu 60\% der Fälle beschrieben.

Bei misslungener Osteosynthese besteht die Möglichkeit der Reosteosynthese mit einem der oben beschriebenen Verfahren. Eine weitere Möglichkeit bei persistierender Bewegungseinschränkung oder Schmerzen ist bei stabilen ligamentären Verhältnissen die Radiusköpfchenresektion. Bei instabiler Situation oder bei Vorliegen einer Essex-Lopresti-Verletzung ist die Indikation zur Versorgung mit einer Radiusköpfchenprothese gegeben.

Die Prothesenimplantation verhindert eine proximale Migration bei rupturierter Membrana interossea, führt zu erhöhter Stabilität und gewährleistet eine bessere Kraftverteilung der von distal wirkenden Kräfte im Ellenbogengelenk.

Eine Radiusköpchenresektion setzt einen stabilen Kapsel-Band-Apparat voraus. Bei Instabilität ist in Abhängigkeit der klinischen und sozialen Situation eine Radiusköpfchenprothese indiziert.

\section{Monteggia-Verletzung}

Die Monteggia-Verletzung ist mit 2-5\% aller proximalen Unterarmfrakturen eine seltene Kombinationsverletzung aus proximaler Ulnafraktur und Luxation des
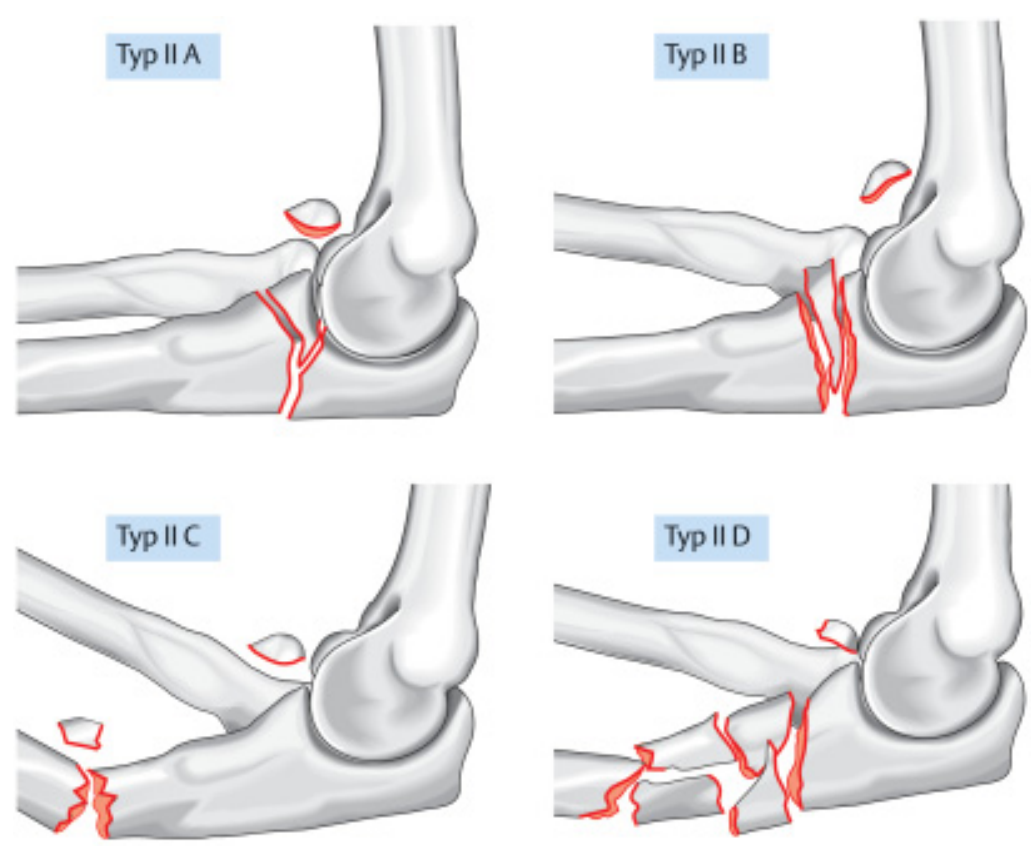

Abb. 7 Subklassifikation der Bado-Einteilung nach Jupiter et al. 1991; entnommen aus Josten C, Lill H. Ellenbogenverletzungen 2002.

Radiusköpfchens [5]. Die Klassifikation erfolgt anhand der Bado-Einteilung. Der häufigste Verletzungstyp ist der Typ II nach Bado. Dieser wird, nach Jupiter [3], noch einmal in 4 Subtypen unterschieden. Die Art der Verletzung steht in direktem Zusammenhang mit der einwirkenden Kraftkomponente. Die Therapie der Wahl bei Monteggia-Verletzungen ist die Plattenosteosynthese und die möglichst rasche und exakte Reposition des luxierten Radiusköpfchens. Eine routinemäßige Rekonstruktion des Lig. annulare scheint nicht notwendig, vielmehr ist zur Prophylaxe von späteren Bewegungseinschränkungen auf eine kurze Immobilisationszeit und eine frühfunktionelle Behandlung zu achten.

Besteht nach Abschluss der primären Behandlung eine bleibende Instabilität im Humeroradialgelenk, muss eine nichtanatomische Rekonstruktion der Ulna ausgeschlossen werden. Ist dies der Fall, sollte eine Reosteosynthese mit entsprechender Korrektur der Ulna erfolgen. Kommt es trotz anatomischer ulnarer Reposition zu einer Instabilität, ist die Resektion des Radiusköpfchens gerechtfertigt und kann je nach Situation mit der Implantation einer Radiusköpfchenprothese kombiniert werden.

In bis zu 5\% der Fälle kommt es bei Monteggia-Verletzungen zur Ausbildung

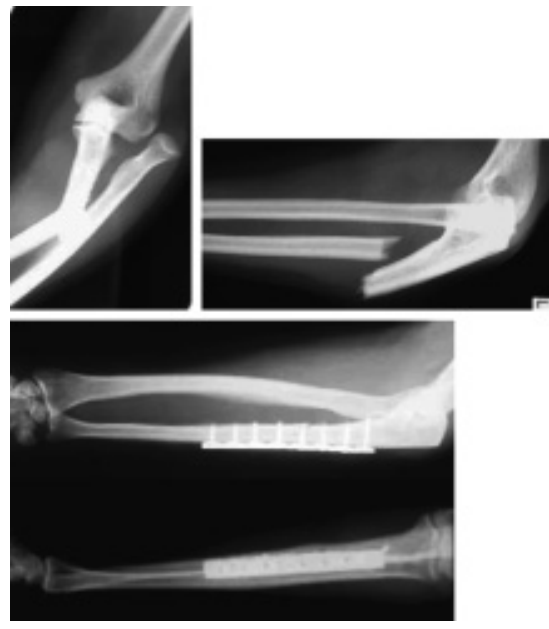

Abb. 8 Osteosynthese einer Monteggiafraktur Typ Ilc die zu einer vollständigen Frakturkonsolidierung mit gutem Bewegungsausmaß führte.

einer radioulnaren Synostose. Damit einhergehende Einschränkung von Pro- und Supinationsbewegungen machen eine chirurgische Abtragung und Einlage eines Interponats notwendig. Postoperativ kann das Risiko einer erneuten Synostosenausbildung mit der systemischen Gabe von NSAR und ggf. einer Bestrahlung reduziert werden.

Kommt es bei Vorliegen einer Mason-IIIFraktur des Radiusköpfchens, nicht refi- 
xierbaren Coronoidfrakturen, einer ausgeprägten Gelenkbeteiligung oder einer ventralen Trümmerzone der Ulna zu einem unbefriedigenden Endergebnis, kann nach individueller Abwägung auch die Implantation einer Ellenbogenprothese als Revisionseingriff gerechtfertigt sein. Insgesamt konnten Korner et al. [4] zwar überwiegend gute funktionelle Ergebnisse nachweisen, jedoch war in $28 \%$ ein Revisionseingriff notwendig.

Eine bleibende humeroradiale Instabilität kann eine Reosteosynthese der Ulna notwendig machen. Bei Instabilität und anatomischer ulnarer Rekonstruktion bietet die Resektion des Radiusköpchens ggf. in Kombination mit einer Radiuskopfprothese eine weitere Therapieoption.

\section{Literatur}

${ }^{1}$ Bauer GJ, Hoellen I. Die distale intrartikuläre Humerusfraktur. Diagnostik, Operationstechnik und Ergebnisse. Aktuelle Traumatologie 1997; 27: 125 -131

2 Beck E. Konservative Behandlung von Brüchen am distalen Oberarmende. Hefte Unfallheilk 1982; 155: 26-34

${ }^{3}$ Jupiter JB, Leibovic SJ, Ribbans W, Wilk RM. The posterior Monteggia lesion. J Orthop trauma 991; 5: $295-402$

${ }^{4}$ Korner J, Hoffmann A, Rudig L, Müller LP, Hessmann M, Lill H, Josten C, Rommens PM. Monteggia injuries in adults: Critical analysis of injury pattern, management and results. Der Unfallchirurg 2004 (online-Publikation)

${ }^{5}$ Josten C, Lill H (Hrsg) Ellenbogenverletzungen. Steinkopff, Darmstadt, 2002

${ }^{6}$ Mason ML. Some observations on fractures of the head of the radius with a review of one hundred cases. Br J Surg 1954; 42: $123-132$

${ }^{7}$ McKee MD, Jupiter LB. Trauma to the adult elbow and fractures of the distal Humerus. In: Browner BD, Jupiter LB, Levine AM,. Trafton PG (eds) Skeletal trauma. Saunders, Philadelphia, 1998; pp 383-404

8 Müller ME, Allgöwer M, Schneider R, Willenegger $\mathrm{H}$. Manual der Osteosynthese. Springer Berlin Heidelberg New York, 1992

9 Schatzker J. Olecranon fractures. In: Schatzker J, Tile M. (eds) The rationale of operative fracture care. Springer, 1987; pp 80-87

${ }^{10}$ Seiler H, Trentz O. Bikondyläre Frakturen. Orthopäde 1988; 17: 262-271
Prof. Dr. med. Christoph Josten Ärztlicher Direktor

Dr. med. Bastian Marquaß

Assistenzarzt Unfallchirurgie

Universitätsklinikum Leipzig

Abteilung für Unfall- und

Wiederherstellungs- und Plastische

Chirurgie

Liebigstraße 20

D-04103 Leipzig 